\title{
RSSI WSN localization with Analysis of Energy Consumption and Communication
}

\author{
S.B. Kotwal \\ SMVD University, Katra, \\ J\&K-182320 India
}

\author{
Shekhar Verma \\ IIIT, Allahabad \\ UP-211012 India
}

\author{
R.K. Abrol \\ SMVD University, Katra, \\ J\&K-182320 India
}

\begin{abstract}
Distance based localization techniques have always been of interest among researchers. The free received signal strength index (RSSI) requires no extra hardware for distance measurement. Authors in this paper assume that RSSI based distance estimation technique will have some error due to noisy RSSI readings. The localization algorithm proposed here takes into account this error and localizes a WSN in three stages. Due to this error in distance, nodes in neighbor of three anchor nodes determine their uncertainty region with some accuracy and become virtual anchors. These nodes then help other nodes in the network to determine their region. These non virtual anchor nodes collaborate among themselves to further decrease the size of uncertainty region. The collaborative nature of nodes is exploited to increase the accuracy and precision of localization. The authors in this paper have used only three anchor nodes to localize a full blown Wireless Sensor Network (WSN) of 100 nodes with better accuracy compared to existing techniques using RSSI till date. Authors also analyze the energy and communication cost involved in localization process.
\end{abstract}

\section{Keywords}

RSSI, WSN localization, Virtual Anchors, Less Communication, Low power Localization.

\section{INTRODUCTION}

With the ever increasing applications of WSN, the need for robust localization technique has also arisen. Localization plays an important role in the key application areas of Wireless Sensor Networks (WSN). For typical applications like road traffic monitoring, health monitoring, efficient routing, target tracking, inventory management, remote environment surveillance, it is hard to image that information will be of real use if not tagged with location information. WSN localization is estimation of the locations of sensors with reference to some local or global coordinate system by using knowledge of inter-sensor node measurements like distance and/or angle. Some localization techniques do not even require these measurements; like hop count [1], [2], APIT [3] and bounding box. But these localization techniques are very prone to errors due to obstructions [4] in the deployment region and are therefore least accurate.

For a WSN comprising of few nodes, individual locations can be programmed manually. Nevertheless when the number of nodes in a WSN is in hundreds or thousands or nodes are placed randomly in a hostile environment; an automated procedure for localization of nodes is required. Since the localization is a vital operation in WSN, therefore any solution proposed for localization has to take accuracy and efficiency as performance metrics. For localization to begin, few sensor nodes with known location information are required and are known as anchors. Their locations of anchor nodes can be determined by using a global positioning system
(GPS), or by placing these anchors at points with known coordinates. But use of GPS equipment on all nodes is not feasible as localization is not the only goal of a WSN application, it is a step in the formation of WSN and using costly GPS equipment on each node would add to the hardware and energy cost which doesn't gel with the idea of wireless sensor networks having small, low energy and low cost sensor nodes for efficient sensing and communication. Detailed issues related to the use of GPS have been already been discussed in [5], [6], [7]. Considering all these issues, GPS cannot be proposed to be used for all the nodes in a network.

\section{RELATED WORK}

A number of localization schemes have been proposed so far which rely on some inter-node measurements for further computation of location of unknown nodes. Since the work proposed is based on RSSI distance approximation, therefore the related work presented here primarily focuses on distance based localization schemes. The limit of localization accuracy is given by Cramer Rao lower bound (CRLB). As per [8] using RSSI for distance measurement, the CRLB of accuracy is $50 \%$ of average inter-node distance, which is acceptable for most of the outdoor applications but for an indoor localization, this error may be large. Most of the localization schemes using RSSI have been able to be more accurate than CRLB and are discussed below.

The localization schemes can be classified on the basis of different aspects like:

a) Centralized vs. distributed,

b) Anchor based vs. Anchor less and

c) Range based vs. Range free

The authors in [9], presented an MDS-MAP based centralized algorithm for computing the coordinates of unknown nodes after approximating the distances between the nodes using shortest path algorithm, whereas in [10], a centralized minimum least square approximation (LS) method is used for localization after distance approximation using RSSI measurements. Scheme proposed in [9] has localization error of $50 \%$ or radio range when distance error is $20 \%$ of it and [10] has $45 \%$ of error with respect to the average node distance. In [11] using GA, the location error has decreased below $25 \%$ of the transmission range but requirement of number of anchor nodes has increased significantly adding to the cost of the system. The distributed algorithm in [12] was able to decrease the error significantly up to $6 \mathrm{~cm}$, but at the cost of using mobile nodes and acoustic energy for distance approximation. In [13] the authors have used Kalman filter based distributed localization algorithm to determine the location of nodes in WSN with mean error less than $28 \mathrm{~mm}$, but the simulation parameters assume the Gaussian noise contributing to the measurement error is less that $1 \%$ of the radio range. The distance measurement techniques till date have not been accurate so far. In most of the applications the 
global coordinates are rather preferred than just local coordinates therefore, anchor based localization has been in focus recently.

In [14], box type distance based localization scheme is implemented. The error of localization with more than three anchors in a deployment region of $100 \mathrm{~m} \times 100 \mathrm{~m}$ with distance error equal to $20 \%$ of communication range is nearly equal to $1.7 \mathrm{~m}$ and variance $1.3 \mathrm{~m}^{2}$ respectively. Centroid based algorithms [15] estimate the location of a sensor by calculating the centroid of anchors within communication range. These types of techniques require sufficiently large no. of anchors as at least three anchors are required in the vicinity of each unknown node to approximate its location. This also requires a large number of anchors. In other range free techniques like APS[16] and Hop-TERRAIN, the no. of anchors required are less but there is small error in localization only when the shortest paths between anchors and unknown nodes approximate to their actual distances. Out of the various range free anchor based localization schemes [15], [16], [17], [18] the anchor based localization scheme proposed in [17] is a range free and gives birth to the concept of a new term called virtual anchors. The scheme utilizes the reliable minimal hop length as the threshold to differentiate between reliable anchors and unreliable anchors.

In contrast to anchor based localization schemes, the anchor less schemes require inter node distance measurement for creating a local map of the nodes. The local map created is not a unique one and can be stitched to any coordinate system with the help of translation, rotation or flipping. The MDSMAP scheme like in [9] can create a local map of the nodes in WSN without anchors but at least three anchors would be required to create a global map without flip ambiguity problem. Authors in [21] have proposed to use multiple power levels to determine distance range and collaborative communication between nodes in a multi-anchor scenario to localize a WSN

All the schemes discussed above require more than three anchors for localization error within acceptable limit for indoor or outdoor localization. The communication cost is a major factor deciding the lifetime of small sized sensor nodes. Most of the schemes have not taken communication cost as a metric of performance of localization scheme.

\section{PROBLEM STATEMENT}

Due to various factors as discussed before, the distance measured between two nodes using RSSI has some error associated with it. The error in actual and estimated location coordinates $\left(\mathrm{x}_{1}, \mathrm{y}_{1}\right)$ and $\left(\mathrm{x}_{2}, \mathrm{y}_{2}\right)$ of an unknown node respectively can be calculated as:

$$
e=\left|d-\sqrt{\left(x_{1}-x_{2}\right)^{2}+\left(y_{1}-y_{2}\right)^{2}}\right|
$$

In [19], the approximate error in distance measurement using RSSI is shown $=1.5 \mathrm{~m}$ for range $>15 \mathrm{~m}$ indoors and $5 \mathrm{~m}$ for range of $100 \mathrm{~m}$ outdoors. Recent advancements in the direction of increasing distance measurement accuracy [20] have shown that using Gauss model for processing RSSI data it is practical to assume that distance error will be within 7 to $17 \%$ of actual distance.

We now formulate the problem that we have tried to solve. Assuming:

a) The inter node distance using RSSI measurement follows Gaussian distribution.

b) All nodes can communicate in duplex mode.

c) At least there is one unknown node within the communication range of three anchor nodes. d) All nodes can compute simple geometric computational algorithm like: intersection, dilation etc. Develop a distributed localization scheme with high accuracy so that $e$ is minimized. The localization scheme should be able to exploit the collaboration within nodes to reduce the error propagation away from anchors and not put burden on network by increasing the communication between nodes as localization is not the only aim of wireless sensor networks.

\section{PROPOSED SOLUTION}

The three anchor nodes A1, A2 and A3 transmit their location to all the nodes in communication range. In a scenario shown in fig-1, there is one unknown node U1 in the communication range of all three anchor nodes and receives the location of these anchor nodes. With RSSI measurement, the distance is approximated with an error of $10 \%$ of actual. The localization algorithm is divided into three stages i.e. populating virtual Anchors, localization using virtual anchors and lastly internode localization.

\subsection{Populating Virtual Anchors}

For all nodes, first step is to determine if they are within communication range of at least three anchors and if yes then these nodes determine their location using trilateration. These nodes which take part in first stage of localization using trilateration from three anchor nodes are named as virtual anchors. For explanation of algorithm, the example of three anchors (A1, A2 and A3) and one virtual anchor (U1) is taken as in fig-1. Using distance measurement from anchor nodes A1 to A3, the U1 node updates its feasibility region as intersection of the deployment area with the circular ring formed around anchor nodes. The node U1's feasibility region is approximated as shown in fig-1.

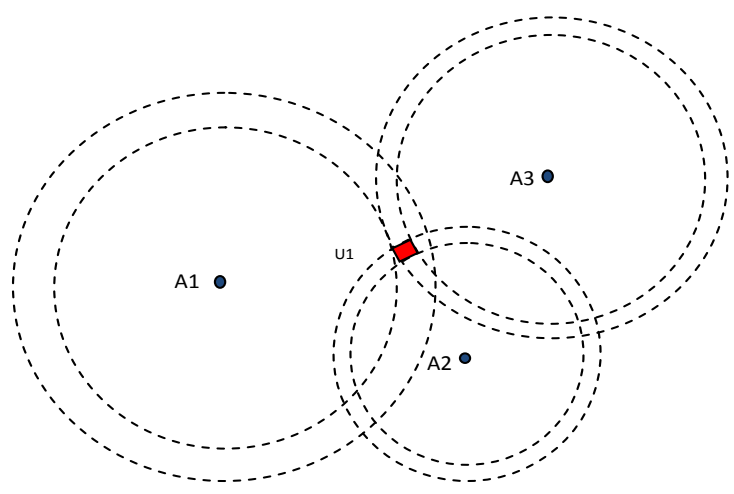

Fig-1.Anchors A1, A2 and A3 help determine the feasibility region (red) of unknown node $U 1$.

These virtual anchors after trilateration assume their position to be at the center of region formed as VA1 in fig- 2 . The advantage of populating virtual anchors is that these nodes have best approximation of their location next to actual anchors and can be used to localize other nodes.

\subsection{Localization Using Virtual Anchors}

At this stage of localization, all other nodes take help of anchor and virtual anchors to determine their location. As shown in fig-2, the node VA1 then transmits its feasibility region's $(x, y)$ coordinates to the other nodes in the communication range. Other nodes which are at one hop from 
at least two anchors and this VA1 determine their own feasibility region using trilateration only.

$\mathrm{U} 2$<smiles>C1CCC1</smiles>

A2

Fig-2: Anchors A1 and A2 with the help of VA1 determine the region of unknown node $\mathrm{U} 2$ and Anchors A2 and A3 with the help of VA1 determine the region of node $U 3$

\subsection{Internode Localization}

After all nodes determine their regions using anchors and virtual anchors, now it is turn of these nodes to collaborate with each other to help reduce their region without using anchors and virtual anchors.
$\mathrm{U} 2$

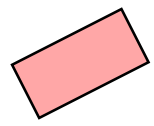

U3

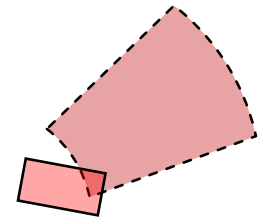

A3

A2
Fig-3: U2 helps U3 reduce its feasibility region.

As shown in fig-3 and 4, the nodes U2 and U3 collaborate and reduce each other's feasibility region.

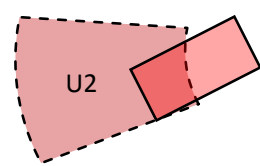

U3

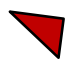

A3 0

A1

VA1

A2

Fig-4: U3 helps U2 reduce its feasibility region.
Node U2 sends its $x, y$ coordinates to the U3 and then U3 updates its region as intersection of old region with the new region w.r.t. U2 are shown in fig-3. This region of U3 helps $\mathrm{U} 2$ to further reduce its region as shown in fig- 4 . The final regions of $\mathrm{U} 2$ and $\mathrm{U} 3$ are shown in fig-5.
U2

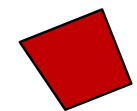

A1 0
VA1
Fig-5: Final regions of $\mathrm{U} 2$ and $\mathrm{U} 3$.

The key to this proposed solution is reduction of error propagation by effective collaboration among nodes. The proposed solution is different from an approach used by authors in [16] as the concept of virtual anchors does not exist there and the localization process there does not seek for at least three anchors in the neighborhood for localization of an unknown. The advantage of virtual anchors is that with limited no. of actual anchors, the total no. of anchors (actual + virtual) is more. This increased no. of anchors helps reduce flip ambiguity which is a prevalent phenomenon and contributes largely to localization error in distance based techniques [18]. Moreover the data packet format in our proposed scheme does not include power level field as it is kept maximum for all nodes and is almost identical for all homogenous nodes, therefore no need to broadcast this information over the network

\section{PERFORMANCE ANALYSIS}

For performance of proposed algorithm it is analyzed in Matlab®, where 100 unknown nodes are deployed in $100 \mathrm{~m} x$ $100 \mathrm{~m}$ region randomly with 3 anchor nodes as shown in fig-5.

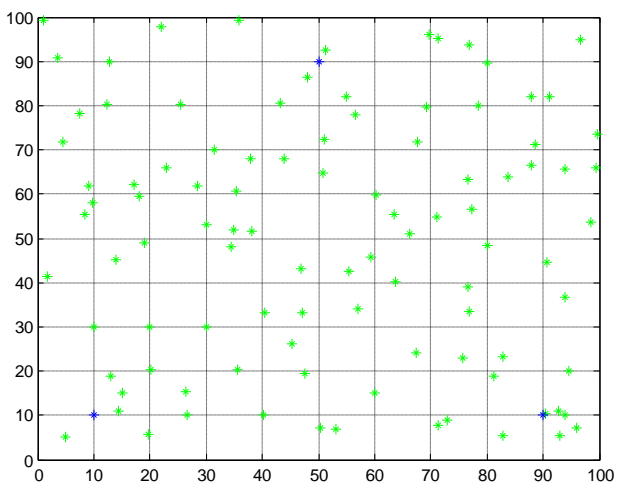

Fig-6: Deployment scenario. 'Green' and 'blue' nodes are unknown anchor nodes respectively. 
All nodes can communicate with other if distance between them is smaller than range $\mathrm{R}=30 \mathrm{~m}$ except anchor nodes, which have communication range up to $70 \mathrm{~m}$. This extended communication range of anchors is assumed because anchor nodes are more powerful in terms of resources when compared to other nodes..The error in distance estimation using RSSI is assumed to be from 5\% to $30 \%$ of R, which is a quite practical range compared to the previous studies [15], [17], [18].

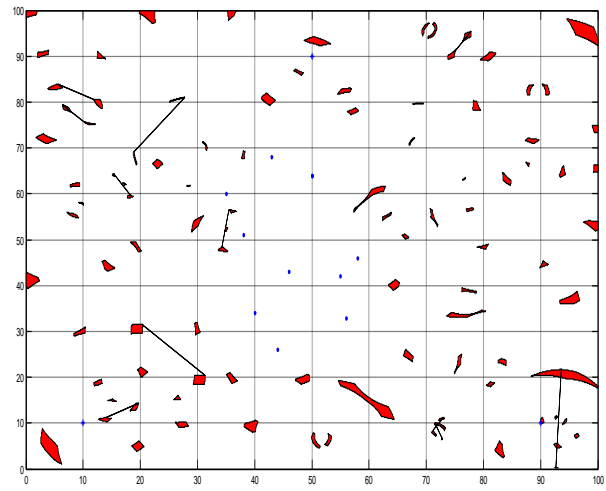

Fig-7: Feasibility regions of unknown nodes after 3 iterations

The proposed algorithm is run for three iterations and after it the regions of unknown nodes are shown in fig-7.

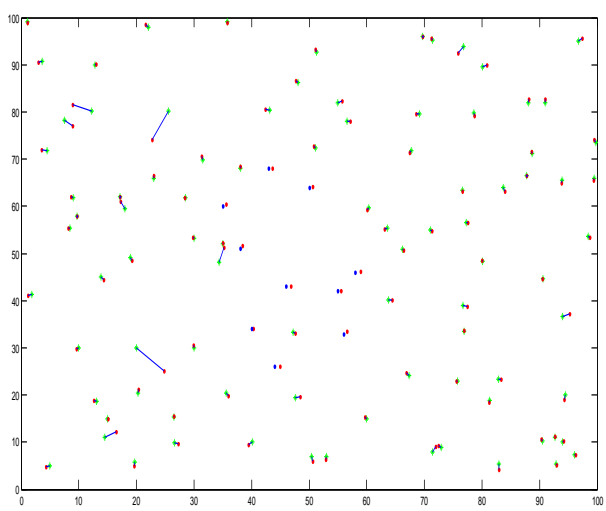

Fig-8: Estimated 'red' vs. actual 'green' locations.

It is worth noting that there are few nodes like one near to (20, 30 ), which do not have a singular feasibility region.

The cause of this flip ambiguity is non availability of three non collinear nodes in the communication range and has already been discussed in detail in [18].

After the nodes have their final regions, their singular ( $\mathrm{x}, \mathrm{y})$ coordinates are approximated as mean of all coordinates. The final locations assumed as mean of feasibility regions of all unknown nodes are shown in comparison to the actual locations in fig-8. The nodes with flip ambiguity have the more error of localization compared to other nodes. This flip ambiguity can further be eliminated by using nodes with higher density.

\subsection{Localization Error vs. Iteration}

Localization error is calculated using (i). There is significant decrease in error from $1^{\text {st }}$ to $3^{\text {rd }}$ iteration, but in $4^{\text {th }}$ and $5^{\text {th }}$ iteration, there is not much improvement.

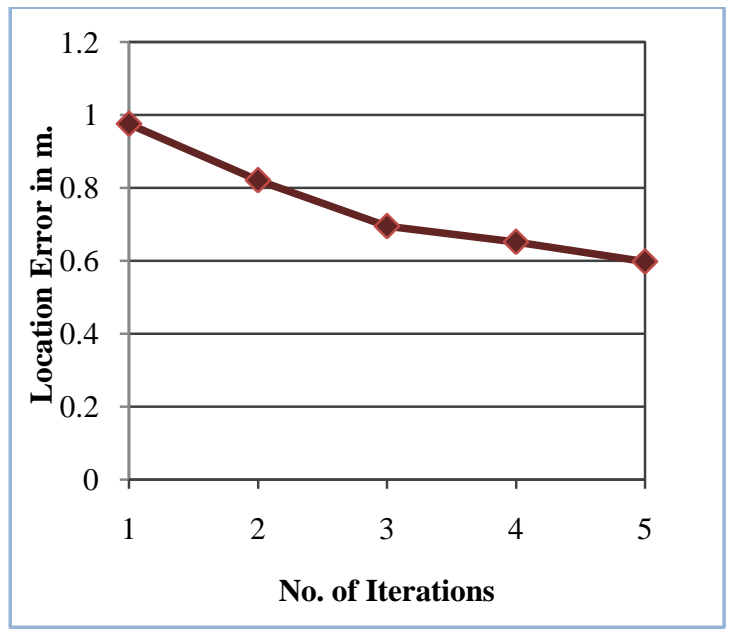

Fig-9: Mean Localization Error per Iteration.

This is because, error reduction after a fixed value is not feasible due to flip ambiguity caused in some of the nodes as shown in fig- 9 .

\subsection{Localization Error vs. Distance Error}

Table-1 below shows the relationship between error in distance vs. localization error and standard deviation.

Table-1. Relation between localization error vs. ranging error.

\begin{tabular}{|c|c|c|}
\hline $\begin{array}{c}\text { Error in } \mathbf{R} \\
\text { as } \% \text { of } \mathbf{R}\end{array}$ & $\begin{array}{c}\text { Mean error in } \\
\mathbf{~}\end{array}$ & S.D. of $\mathbf{e}$ in $\mathbf{~}$ \\
\hline $\mathbf{5}$ & 0.53 & 0.88 \\
\hline $\mathbf{1 0}$ & 0.7 & 0.84 \\
\hline $\mathbf{1 5}$ & 1.09 & 0.99 \\
\hline $\mathbf{2 0}$ & 1.33 & 1.81 \\
\hline $\mathbf{2 5}$ & 1.52 & 1.21 \\
\hline $\mathbf{3 0}$ & 1.58 & 2.50 \\
\hline
\end{tabular}

It is clear from the table- 1 that with the increase in ranging error from $5 \%$ to $30 \%$ of $\mathrm{R}$, the localization error increases significantly, but the maximum mean error with $30 \%$ of $\mathrm{R}$ is nearly $1 \mathrm{~m}$ and $53 \mathrm{~cm} \mathrm{~cm}$ in $5 \%$ error of R, which is comparable to the localization techniques proposed in [9],[10],[12],[13],[14] and [17]in such scenario.

\subsection{Analysis of Energy Consumption and Communication Cost}

The total power required is a sum of both the power required to transmit messages and the power required to receive (or process) messages. We determine the no. of messages transmitted and received for localization process. Excluding 
anchor and virtual anchor communications which are very less, an unknown node $\mathrm{k}$ transmits its ID and Uncertainty region coordinates to all the $\mathrm{N}_{\mathrm{k}}$ nodes in its communication range. Therefore the total transmissions and receptions by kth node are equal to $\mathrm{N}_{\mathrm{k}}$ and total messages transmitted on network will be given by:

$$
\mathrm{N}_{\mathrm{T}}=\sum_{\mathrm{k}=1}^{\mathrm{M}} \mathrm{N}_{\mathrm{k}}
$$

Where $\mathrm{M}$, is the total number of unknown nodes in the network excluding anchors and virtual anchors.

Assuming power required for transmission and reception of a message packet is equal to $\mathrm{P}_{\mathrm{Tx}}$ and $\mathrm{P}_{\mathrm{Rx}}$ respectively, the total energy required for communication will be:

$$
\mathrm{P}_{\mathrm{T}}=\sum_{\mathrm{k}=1}^{\mathrm{M}} \mathrm{N}_{\mathrm{k}}\left[\mathrm{P}_{\mathrm{Tx}}+\mathrm{P}_{\mathrm{Rx}}\right]
$$

Where $\mathrm{P}_{\mathrm{Tx}}+\mathrm{P}_{\mathrm{Rx}}=137 \mathrm{~mW}$ [23], if supply voltage is assumed to be $3.6 \mathrm{~V}$ and current consumption for Tx mode and $\mathrm{Rx}$ mode nearly equal to $19 \mathrm{~mA}$. Therefore eqn. (iii) becomes:

$$
\mathrm{P}_{\mathrm{T}}=274 \sum_{\mathrm{k}=1}^{\mathrm{M}} \mathrm{N}_{\mathrm{k}}
$$

Same modeling for communication can be done for other types of localization algorithms; therefore it can be assumed fairly that the energy consumption by proposed technique is equivalent to other distance based localization techniques including [21]. Moreover we propose that once localization process is complete, the node ID can be replaced by its $\mathrm{x}, \mathrm{y}$ coordinates to save energy further.

\section{CONCLUSION}

A localization technique with inaccurate distance information from noisy RSSI values is presented. The nodes collaborate with each other to reduce error propagation. It is observed that most of the error is reduced in first iteration of algorithm only. To the best knowledge of authors, the mean error is less than any other existing technique till date. The communication cost per iteration is also comparable to the existing techniques. At the same time it was observed that the mean error and area reduction per iteration is not significant after $3^{\text {rd }}$ iteration. Proposed algorithm has also shown that using only three anchors, the desired accuracy of few centimeters is possible with 3 iterations of the algorithm making it suitable for indoor localization applications.

\section{REFERENCES}

[1] D. Niculescu and B. Nath, "DV Based Positioning in Ad Hoc Networks", presented at Telecommunication Systems, 2003, pp.267-280

[2] C. Savarese, J. Rabaey, and K. Langendoen, "Robust positioning algorithms for distributed ad-hoc wireless sensor networks", in the Proc. General Track: 2002 USENIX Annual Technical Conference, 2002, pp.317327.

[3] Jia Wang and Fu Jingqi, "Research on APIT and Monte Carlo Method of Localization Algorithm for Wireless Sensor Networks". LSMS/ICSEE (2) 2010: 128-137.

[4] Y. Zeng, S. Zhang, S. Guo, and X. Li, "Secure HopCount Based Localization in Wireless Sensor Networks", in Proc. CIS, 2007, pp.907-911.
[5] Akgul, Ferit Ozan, Mohammad Heidari, Nayef Alsindi, and Kaveh Pahlavan. "Localization Algorithms and Strategies for Wireless Sensor Networks: Monitoring and Surveillance Techniques for Target Tracking". Localization Algorithms and Strategies for Wireless Sensor Networks: Monitoring and Surveillance Techniques for Target Tracking. IGI Global, 2009. 5495.

[6] Guibin Zhu, Qiuhua Li, Peng Quan and Jiuzhi Ye, "A GPS-free localization scheme for wireless sensor networks," in Proc. Communication Technology (ICCT) 2010 12th IEEE International Conference on vol., no. pp.401-404, 11-14 Nov. 2010.

[7] Arthi, R. and Murugan, K., "Localization in Wireless Sensor Networks by Hidden Markov Model," in Proc. Advanced Computing (ICoAC), 2010 Second International Conference on , vol., no., pp.14-18, 14-16 Dec. 2010.

[8] Qi, Y and Kobayashi, H. "On relation among time delay and signal strength based geolocation methods". In Proc. IEEE Global Telecommunications Conference. GLOBECOM' 03. 7, 4079-4083.

[9] Y. Shang, W. Ruml, Y. Zhang, and M. Fromherz, "Localization from mere connectivity", In Proc. of ACM Symposium on Mobile Ad Hoc Networking and Computing (MobiHoc'03), June 2003, Annapolis, Maryland, USA, pp. 201-212.

[10] Cesare Alippi and Giovanni Vanini, “A RSSI-based and calibrated centralized localization technique for Wireless Sensor Networks", in Proceedings of Fourth IEEE International Conference on Pervasive Computing and Communications Workshops (PERCOMW'06), Pisa, Italy, March 2006, pp. 301-305.

[11] Q. Zhang, J. Huang, J. Wang, C. Jin, J. Ye, W. Zhang, 2008, "A new centralized localization algorithm for wireless sensor network," In Proc. of the IEEE ChinaCom 2008, pp. 625--629.

[12] ZHONG, Zhi LUO, Da-Yong LIU, Shao-Qiang FAN, Xiao-Ping QU, Zhi-Hua, "An Adaptive Localization Approach for Wireless Sensor Networks Based on Gauss-Markov Mobility Model". Acta Automatica Sinica, 36 (11), p.1557, Nov 2010

[13] A. Savvides, H. Park, and M. Srivastava, "The bits and flops of the n-hop Multilateration primitive for node localization problems", In Proceedings of the 1st ACM international Workshop on Wireless Sensor Networks and Applications (WSNA'02), September 2002, Atlanta, Georgia, USA, pp. 112-121.

[14] Mourad F, Snoussi H, Abdallah F and Richard C, "Anchor-Based Localization via Interval Analysis for Mobile Ad-Hoc Sensor Networks," Signal Processing, IEEE Transactions on , vol.57, no.8, pp.3226-3239, Aug. 2009.

[15] N. Bulusu, J. Heidemann, and D. Estrin, "GPS-Less Low Cost Outdoor Localization for Very Small Devices," IEEE Personal Comm. Magazine, vol. 7, no. 5, pp. 2834 , Oct. 2000

[16] D. Niculescu and B. Nath, "Ad Hoc Positioning System," Proc. IEEE GLOBECOM, pp. 2926-2931, Nov. 2001.

[17] B. Xiao, L. Chen, Q. Xiao, and M. Li, "Reliable anchorbased sensor localization in irregular areas," accepted in IEEE TMC, 2009. 
[18] C. Savarese, J. Rabaey, and K. Langendoen, "Robust Positioning Algorithm for Distributed Ad Hoc Wireless Sensor Networks," in Proc. USENIX Ann. Technical Conf., pp. 317-327, June 2002.

[19] S. Lanzisera, K. Pister, "RF Ranging Methods and Performance Limits for Sensor Localization," in Localization Algorithms and Strategies for Wireless Sensor Networks: Monitoring and Surveillance Techniques for Target Tracking. G. Mao, B. Fidan, IGI Global, 2009.

[20] Zhang Jianwu, Zhang Lu, "Research on distance measurement based on RSSI of ZigBee", CCCM 2009. ISECS International Colloquium on , vol.3, no., pp.210212, 8-9 Aug. 2009.
[21] M.L. Sichitiu, V. Ramadurai, and P. Peddabachagari, "Simple Algorithm for Outdoor Localization of Wireless Sensor Networks with Inaccurate Range Measurements", in Proc. International Conference on Wireless Networks, 2003, pp.300-305.

[22] A. A. Kannan, B. Fidan, and G. Mao, "Analysis of Flip Ambiguities for Robust Sensor Network Localization," IEEE Transactions on Vehicular Technology, vol. 59, no. 4, pp. 2057-2070, May 2010.

[23] Chipcon 2240 data sheet at "www.focus.ti.com/lit/ds/symlink/cc2420.pdf" $14^{\text {th }}$ June2012. 